\title{
On the origin of time-dependent behaviour in a barotropically unstable shear layer
}

\author{
W.-G. Früh ${ }^{1}$ and A. H. Nielsen ${ }^{2}$ \\ ${ }^{1}$ Department of Mechanical and Chemical Engineering, Heriot-Watt University, Riccarton, Edinburgh, EH14 4AS, UK \\ ${ }^{2}$ Association EURATOM-Risø National Laboratory, Optics and Fluid Dynamics Department, DK-4000 Roskilde, Denmark
}

Received: 5 August 2002 - Accepted: 15 January 2003

\begin{abstract}
An experimental study on the instability of a detached Stewartson layer, using an annular, rotating tank with flat, rigid upper and lower boundaries, showed an instability to steady vortices at a critical Reynolds number, arranged in a global mode structure along the shear layer. Increasing the Reynolds number resulted in successive transitions to lower modes where time-dependent behaviour was only found for flows with three or less vortices. Previous numerical simulations of a related experiment, using a two-dimensional spectral model of the quasi-geostrophic vorticity equation incorporating Ekman forcing and viscous dissipation, suggested that the boundary conditions at the inner cylinder of the domain could significantly affect the interior flow by the generation and shedding of vorticity at this inner boundary. A comparison of the numerical results with experimental data suggests that the rise of time-dependent behaviour is due to vorticity generation at the inner domain boundary.
\end{abstract}

\section{Introduction}

Rotating shear layers occur in a variety of situations, from industrial to geophysical applications. An example of an industrial application is the design of computer hard disk drives (Humphrey and Gor, 1993) where shear layers occur between the rapidly rotating magnetic disc and the stationary housing. It has been proposed that vortical features on the giant planets, such as the Great Red Spot on Jupiter, are a result of this instability, e.g. Meyers et al. (1989). On an intermediate scale, the breakdown of a weather front can be explained as the instability of the front due to the velocity shear across the front (Schär and Davies, 1990).

One of the main features of rotating flows is the generation of large-scale coherent structures whose evolution plays a major role in the overall behaviour and predictability of geophysical flows. The system studied follows the classical split-disk forcing on an $f$-plane, first considered by Stewart-

Correspondence to: Früh (w.g.fruh@hw.ac.uk) son (1957); a fluid of depth $H$ and with a background rotation of $\Omega$ is contained between two horizontal boundaries.

Compared to shear layers in non-rotating fluids, the effects of a background rotation are two-fold. For one, a strong Coriolis force inhibits motion aligned with the rotation axis, and the flow tends to be two-dimensional and perpendicular to the rotation axis. This effect can be quantified by the Rossby number, e.g. Pedlosky (1987). An infinitely small Rossby number leads to the geostrophic approximation and purely two-dimensional flow, while a sufficiently small Rossby number (strictly speaking $R o \ll 1$, but even $R o \sim 1$ may in cases be sufficient) indicates the possibility of using the quasi-geostrophic approximation.

The second effect is that a shear region, for example due to an imposed velocity jump, is confined to a shear layer which becomes thinner as the background rotation becomes stronger. This scaling of the shear layer will obviously affect the shear instability of a fluid since the definition of the Reynolds number of the flow includes a length scale such as the shear layer thickness. The physical mechanism controlling the extent and structure of the shear layer arises from the presence of Ekman layers which cause a small vertical mass transport in the fluid interior. Continuity requires this mass transport to be balanced, which results in a vertical return flow where two Ekman layers of different mass transport join (Greenspan, 1968, Sect. 2.18). One of the factors influencing Ekman layers, and consequently the shear layers, is the geometry of the boundaries. In the simplest case of a uniform layer of fluid with horizontal boundaries and vertically uniform forcing of the shear flow as indicated in Fig. 1, Stewartson (1957) has shown that the thickness of the shear layer is proportional to $\Omega^{-1 / 4}$ or $E^{1 / 4}$, where $\Omega$ and $E$ are the background rotation and the Ekman number, respectively. These shear layers are known as Stewartson layers.

Above a critical shear, the Stewartson layer becomes linearly unstable with respect to wave-like perturbations, where the most unstable mode number depends largely on the thickness of the shear layer. The flow is similar to that arising from a Kelvin-Helmholtz instability in that a string of steady 


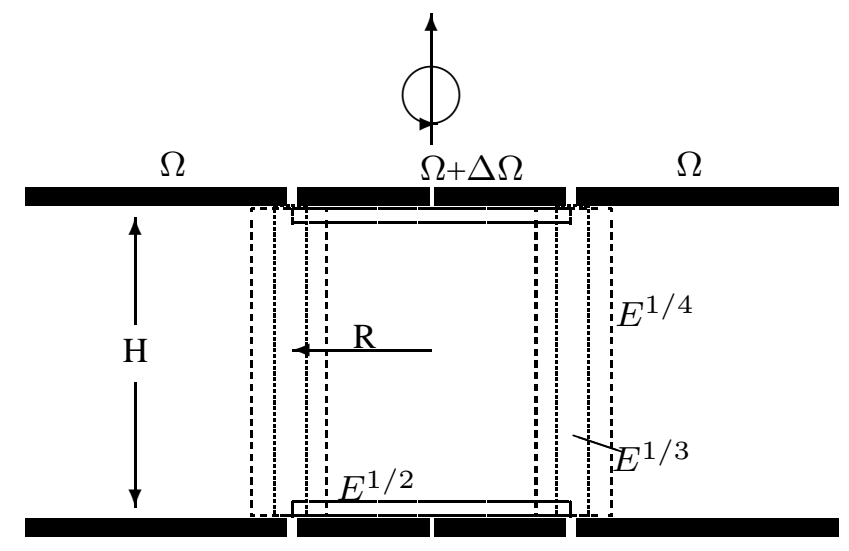

Fig. 1. Stewartson layers after Stewartson (1957).

vortices form in the shear layer, arranged in a regular wavelike pattern. The resulting wave, or vortex flow is still predominantly two-dimensional and can be successfully modelled using the quasi-geostrophic potential vorticity equation. As is common in two-dimensional flows, increasing the forcing results in a succession of mode transitions to lower mode numbers due to nonlinear interactions. Modelling and understanding subsequent bifurcations to timedependent or more complex flows is much more difficult. Some apparently simple situations, such as amplitude vacillation in baroclinic flows, can seemingly be reproduced by low-order quasi-geostrophic models (Früh, 1996) but even then doubt remains as to whether the oscillations observed in the numerical models arise from the same processes as those found in laboratory experiments. Other oscillations, such as structural vacillations, still seem to be fairly organised and regular flows but are not consistent with low-dimensional dynamics (Früh and Read, 1997) and may require ageostrophic dynamics and possibly fully three-dimensional models.

Many experimental studies have investigated different aspects of detached shear layers in rotating fluids using a range of geometries and forcing methods, initiated in a study by Hide and Titman (1967), who suspended a differentially rotating disk in a rotating cylindrical tank with a flat rigid lid. Other experiments, e.g. Antipov et al. (1986) or Nezlin et al. (1990), used thin layers of fluid with a free surface in rotating parabolic vessels with differentially rotating lower boundaries. In addition to mechanical forcing by moving the boundaries, a set of sources and sinks can also be used to establish a velocity shear, e.g. Holton (1971) and Solomon et al. (1993).

The geometry and forcing, which follows most closely Stewartson's (1957) initial work, uses two horizontal rigid boundaries rotating around a common vertical axis of rotation. Rabaud and Couder (1983) and Chomaz et al. (1988) used exactly Stewartson's geometry (bar the necessary side walls) but restricted the forcing to $\Omega \ll \Delta \Omega$ and a shallow layer, which had the effect that the Rossby number was relatively large and that viscosity was important everywhere in the fluid $(E>1)$. Niino and Misawa (1984) and
Früh and Read (1999), on the other hand, chose parameters which resulted in relatively thin Ekman layers $(E \ll 1)$ and fairly small Rossby numbers, a pre-requisite for the quasigeostrophic approximation. Despite these differences in the magnitude of the nondimensional parameters, the observations from the all studies show a very close qualitative agreement, namely an initial well-defined supercritical bifurcation of the axisymmetric flow to steady vortex modes followed by a sequence of decreasing vortex numbers until only few large vortices remained. Time-dependence was observed in both systems only for flows involving three or fewer vortices, and both Chomaz et al. (1988) and Früh and Read (1999) report indications of fully three-dimensional flow regimes.

Only recently has it become feasible to run twodimensional numerical models, let alone fully threedimensional models, at the resolution required to resolve the Stewartson layers in detail even for simple geometries of the flow (Bergeron et al., 2000). The recent generation of twodimensional models can now be used to test hypotheses on the secondary instabilities from the large-scale steady vortices to temporally and spatially more complex flows. Bergeron et al. (2000) succeeded remarkably well in reproducing the observations from the experiments by Rabaud and Couder (1983) and Chomaz et al. (1988) which were dominated by viscosity throughout the entire fluid. In a detailed study, Bergeron et al. (2000) investigated the equilibration of vortices and the dynamics of mode transitions at moderate Reynolds numbers, but also explored high Reynolds numbers where the flow becomes time-dependent. While the side boundaries play only a minor role in the steady flow regimes, they appear to become important for time-dependent flows as a source of vorticity. The same model but with a modified forcing was also successfully applied to a free-surface, shallow-water system in a parabolic annulus, where viscous effects are consigned to relatively thin Ekman and Stewartson layers in the study by de Konijnenberg et al. (1999).

The article is organised as followed: Sect. 2 contains the derivation of the barotropic quasi-geostrophic vorticity equation and a short description of our numerical model, Sect. 3 a description of the experimental set-up, Sect. 4 experimental results of both steady and time-depended flows, Sect. 5 our corresponding numerical results, and finally Sect. 6 contains our conclusions.

\section{The model}

The model is based on the barotropic quasi-geostrophic vorticity equation with forcing due to Ekman pumping and viscous diffusion. The main aspects of the underlying theory, which can be found for example in Pedlosky (1987), Sect. 6.4, are outlined in the next section. This is followed by a description of its implementation in the numerical model. 


\subsection{Theory}

For the derivation of the governing equations, the quantities are scaled by a horizontal velocity scale $U$, a vertical length scale $H$ and a horizontal length scale $L$. Time and horizontal velocities are therefore scaled as $t^{*}=\frac{L}{U} t$ and $u^{*}=U u$, where the starred variables are the dimensional quantities. Consequently, the vertical velocity is scaled by $w^{*}=\frac{D}{L} U w$ and the vertical component of the vorticity by $\omega^{*}=\frac{U}{L} \omega$.

The parameter describing the primary balance of forces in rotating flows is the Rossby number,

$R o \equiv \frac{U}{f L}=\frac{R}{2 L} \frac{\Delta \Omega}{\bar{\Omega}}$,

which is a measure of the nonlinear advection compared to the Coriolis force. $f=2 \Omega$ is the Coriolis parameter, $\Delta \Omega$ and $R$ are the rotation rate and radius of the inner section, respectively. In the case of the split-disk forcing, the velocity scale is the velocity of the edge of the differentially rotating inner circular sections relative to the outer sections, $U=R \Delta \Omega$. The choice of the length scale is somewhat ambiguous; one could either choose a typical scale of the apparatus, for example $L=R / 2$, as Hide and Titman (1967) did, or the thickness of the shear layer which obviously depends on the background rotation and the fluid depth.

In the quasi-geostrophic approximation, the variables in the Navier-Stokes and continuity equations are expanded in powers of the Rossby numbers, e.g. $u=u_{0}+R o u_{1}+$ $R O^{2} u_{2}+\cdots$, where the $O(1)$ terms of the horizontal momentum equations result in the geostrophic flow as the balance of the velocities with the pressure gradients. The $O(R o)$ terms of the equations give the quasi-geostrophic equation. These equations contain the geostrophic velocities in the inertial and viscous terms but the first order corrections of the velocities, $v_{1}$ and $u_{1}$, and of the pressure, $p_{1}$, in the Coriolis terms and pressure gradient terms.

The pressure gradients are eliminated by cross differentiation giving the vorticity equation which then has a term $u_{1_{x}}+v_{1_{y}}$ where the coordinate subscripts denote the respective differentiation. Using the $O(R o)$ continuity equation, this term can be replaced by $-w_{1_{z}}$. In a barotropic model, the horizontal velocities or the vertical component of the vorticity do not vary with height within the fluid interior, $0=u_{1_{x z}}+v_{1_{y z}}=w_{1_{z z}}$. Since in the scaling the vertical co-ordinate is scaled by the fluid depth, $H$, the integration between the bottom, $z=0$, and the top, $z=1$, of the fluid is $w_{1 z}=w_{1, T}-w_{1, B}$.

The effect of viscous dissipation compared to the Coriolis force is measured by the Ekman number,

$E \equiv \frac{v}{\Omega H^{2}}$

where $v$ is the kinematic viscosity of the fluid and $H$ the fluid depth. The presence of horizontal boundaries gives rise to boundary layers, the Ekman layers of thickness $\delta_{E} \propto E^{1 / 2} H$ which affect the interior flow by Ekman pumping. If the lower boundary has a vorticity of, say, $\omega_{B}$, but the fluid outside the boundary layer a vorticity of $\omega$, then the vertical velocity at the top of the boundary layer is

$w_{1, B}=\frac{1}{2} \sqrt{E}\left(\omega-\omega_{B}\right)$.

Similarly, a vorticity $\omega_{T}$ of the upper boundary results in a vertical velocity of

$w_{1, T}=-\frac{1}{2} \sqrt{E}\left(\omega-\omega_{T}\right)$.

Because the case investigated here has identical upper and lower boundaries, the upper and lower boundary conditions can be expressed by a single forcing vorticity, $\omega_{F} \equiv \omega_{B}=$ $\omega_{T}$.

Horizontal dissipation due to horizontal velocity gradients could use a respective horizontal Ekman number using the horizontal length scale, $L$, but it is more common in shear flows to use a Reynolds number, comparing the advection terms to viscous dissipation,

$R e=\frac{U L}{v}$.

The scales to be used are the same as for the definition of the Rossby number, $U=R \Delta \Omega$, and either $L=R / 2$, for example, to define an "external" Reynolds number, or the shear layer thickness to define an 'internal' Reynolds number.

Using the solution for the Ekman pumping in the quasigeostrophic order of the vorticity equation then results in

$\frac{\partial \omega}{\partial t}+\mathbf{u} \cdot \nabla \omega=-\frac{\sqrt{E}}{R o}\left(\omega-\omega_{F}\right)+\frac{1}{R e} \nabla^{2} \omega$.

with the substantial derivative of the geostrophic vorticity on the left hand side and forcing by Ekman layers and viscous diffusion by the horizontal shear on the right hand side, respectively.

Equation (4) contains two dynamically relevant parameters, the Reynolds number $R e$ and the combination of the Ekman and Rossby numbers $E^{1 / 2} R o^{-1}$. The forcing through Ekman pumping depends on the vertical scale, $H$, in the definition of the Ekman number and the horizontal scale, $L$, in the Rossby number while the horizontal internal viscous diffusion seems to depend only on the horizontal length scale used for the definition of the Reynolds number. If the relevant horizontal length scale is that of the thickness of the Stewartson layer, however, then the two length scales become interdependent and all parameters can be reduced to parameters based on one length scale only. According to Niino and Misawa (1984), the Ekman layer thickness in a system with rigid upper and lower boundaries, the Ekman layer thickness is $\delta_{E}=\left(\frac{E}{4}\right)^{1 / 2} H$, and the thickness of the Stewartson layer is $L=\left(\frac{E}{4}\right)^{1 / 4} H$. Inserting $L$ in the definitions of $E^{1 / 2} R o^{-1}$ and $R e^{-1}$, respectively, gives

$\frac{\sqrt{E}}{R o}=\frac{v^{1 / 2}}{\Omega^{1 / 2} H} \frac{2 \Omega L}{U}=\frac{2^{1 / 2} v^{3 / 4} \Omega^{1 / 4}}{H^{1 / 2} U}$ 
and

$$
\frac{1}{R e}=\frac{v}{U L}=\frac{2^{1 / 2} v^{3 / 4} \Omega^{1 / 4}}{H^{1 / 2} U}
$$

respectively. It becomes apparent that the two parameters are not only linked but identical, resulting in the final equation, which can either be written with the geostrophic velocity, $\boldsymbol{u}_{0}$,

$$
\frac{\partial \omega}{\partial t}+\boldsymbol{u}_{0} \cdot \nabla \omega=\frac{1}{R e}\left(\nabla^{2} \omega-\omega+\omega_{F}\right)
$$

or the geostrophic stream function, $\psi$, using

$\nabla^{2} \psi=-\omega$

and the Jacobian,

$J(\omega, \psi)=\frac{1}{r}\left(\frac{\partial \omega}{\partial r} \frac{\partial \psi}{\partial \theta}-\frac{\partial \psi}{\partial r} \frac{\partial \omega}{\partial \theta}\right)$

to give

$$
\frac{\partial \omega}{\partial t}+J(\omega, \psi)=\frac{1}{R e}\left(\nabla^{2} \omega-\omega+\omega_{F}\right)
$$

It might be instructive to return Eq. (7) into dimensional form by multiplying it by $\left(\frac{U}{L}\right)^{2}$, and untangling $E, R o$, and $R e$,

$$
\frac{\partial \omega^{*}}{\partial t^{*}}+J\left(\omega^{*}, \psi^{*}\right)=\frac{2 \sqrt{\nu \Omega}}{H}\left(\omega^{*}-\omega_{F}^{*}\right)+\nu \nabla^{2} \omega^{*} .
$$

Not surprisingly, the forcing term has twice the amplitude of the corresponding equations for the free-surface parabolic annulus as stated, for example, in de Konijnenberg et al. (1999).

\subsection{Numerical model}

The numerical model is that used by, e.g. Bergeron et al. (2000) where more details can be found. It solves Eq. (9) in a polar coordinate system using Fourier-Chebyshev expansion of the solution. The resolution ranges from $256 \times 256$ for low Reynolds numbers up to $1024 \times 1024$ for flows at higher $R e$. The radial resolution is higher near the two solid boundaries with a cosine distribution with radial points $r_{i}$ located at $r_{i}=\cos (\gamma i)$ where $\gamma$ is chosen suitably to cover the radial extent of the domain. As time-stepping a third-order "Stiffly-Stable" scheme was used in which the linear terms are treated implicitly while the nonlinear term, the Jacobian, is treated explicitly. As initial conditions the axisymmetric basic flow of Eq. (9) was used.

It was shown by Bergeron et al. (2000) that the side boundaries play an important role, especially for high Reynolds number, and no-slip boundary conditions at the inner shaft and the outer tank wall were used. The forcing of the fluid is not directly the rotation of the boundaries but a result of Ekman pumping. In the vicinity of the shear layer, the forcing may not be directly given by the disk rotation rates, and a sudden jump corresponding to the edge of the inner disk would not only be a numerical nightmare but also unphysical. Several forms of a suitable forcing function were discussed by Bergeron et al. (2000), and the function chosen for this study was

$v_{\theta}^{F}=\Omega r+\frac{r}{2}\left[1-\tanh \left(\frac{r-R}{\delta_{F}}\right)\right] \Delta \Omega$,

where $v_{\theta}^{F}$ is the azimuthal velocity of the forcing term, e.g. $\omega_{F}=\nabla \times\left(0, v_{\theta}^{F}, 0\right)$. The position of the shear layer is $R$ and the forcing length, $\delta_{F}$, is a measure of the thickness of the shear layer. The mean background rotation, $\Omega$, results in an overall rotation of the solution but does not affect the dynamics. To satisfy the CFL criterion, by which information should not travel in one time step further than the spatial resolution, velocities should be kept small. For time steps as large as possible, a value of $\Omega$ was chosen for which the angular velocity of the resulting vortices is expected to be as small as possible.

The axisymmetric solution, which represents the quasigeostrophic $E^{1 / 4}$-Stewartson layer, depends on the choice of $\delta_{F}$. The solution has a shear region which is much broader than $\delta_{F}$ due to the action of viscous diffusion. Because the shear layer thickness is not precisely known, the prescribed forcing length, $\delta_{F}$, is the natural choice for the Reynolds number for the characterisation of the numerical simulations,

$R e=\frac{R \Delta \Omega \delta_{F}}{v}$.

To match the forcing function to the thickness of the stable shear layer at the Ekman number, $E=10^{-4}$, the forcing length was determined to be $\delta_{F}=9.6 \mathrm{~mm}$. For a direct comparison of the experimental results with the numerical model in the present study, a suitable factor had to be estimated from a comparison of two experimental observations in the stable regime in our apparatus at $E=7 \times 10^{-4}$ and $E=3 \times 10^{-3}$ with the axisymmetric solution of the model. The general scaling of the experimental stable shear layer with $E^{1 / 4}$ is supported by Baker (1967). This comparison suggested that $L=(E / 12)^{1 / 4} H$ was a reasonable number, leading to our definition of the Reynolds number as

$R e \equiv \frac{U L}{v}=12^{-1 / 4}\left(\frac{\Omega H^{2}}{v}\right)^{3 / 4} \frac{R}{H} \frac{\Delta \Omega}{\Omega}$.

\section{The apparatus}

The fluid was contained in a cylindrical perspex tank with a radius of $300 \mathrm{~mm}$ as shown in Fig. 2. A vertical axis of radius $31.8 \mathrm{~mm}$ in the centre of the tank supported two circular disks with a radius of $150 \mathrm{~mm}$ each (the dark-green sections in Fig. 2). Two flat rings were placed in the tank flush with the circular sheets to ensure a uniform fluid depth throughout the domain. The height of the upper disk and ring above the lower surface could be adjusted from 30 to $150 \mathrm{~mm}$ but time constraint did not allow to test different fluid depths and the fluid depth was $100 \mathrm{~mm}$ for all experiments. The inner axis 
Table 1. Physical parameters of experimental rig and of numerical model configuration

\begin{tabular}{|c|c|c|c|c|c|c|c|c|}
\hline \multicolumn{3}{|c|}{ geometry } & \multicolumn{4}{|c|}{ forcing } & \multicolumn{2}{|c|}{ kinematic viscosity } \\
\hline$H$ & $=100$ & $\mathrm{~mm}$ & $\Omega$ & $=$ & $0 \ldots 4$ & $\mathrm{rad} / \mathrm{s}$ & PIV: & $1.66 \times 10^{-6} \mathrm{~m}^{2} / \mathrm{s}$ \\
\hline$R$ & 150 & $\mathrm{~mm}$ & $\Delta \Omega$ & $=$ & $-0.7 \ldots 0.7$ & $\mathrm{rad} / \mathrm{s}$ & LDA: & $10^{-6} \mathrm{~m}^{2} / \mathrm{s}$ \\
\hline$A$ & $=300$ & $\mathrm{~mm}$ & $\delta_{F}$ & $=$ & 9.6 & $\mathrm{~mm}$ & Model: & $0.82 \times 10^{-6} \mathrm{~m}^{2} / \mathrm{s}$ \\
\hline
\end{tabular}

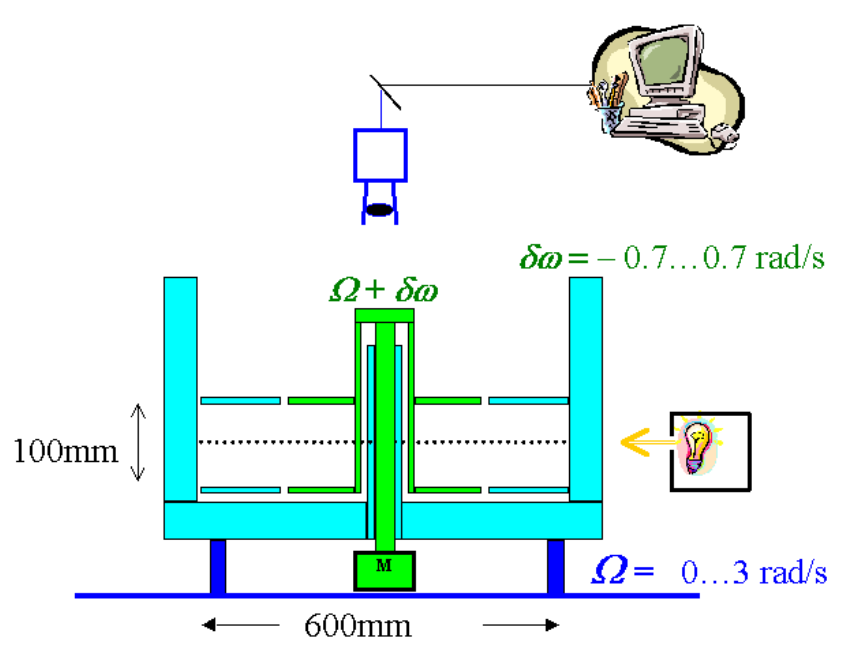

Fig. 2. Schematic diagram of the apparatus.

with the disks was driven by a stepper motor at angular velocities $\Delta \Omega$ ranging from -0.7 to $+0.7 \mathrm{rad} \mathrm{s}^{-1}(\approx \pm 0.1 \mathrm{~Hz})$. The whole tank was mounted on a steel turntable with a diameter of $1.2 \mathrm{~m}$ which rotated anticlockwise at angular velocities up to $\Omega=4 \mathrm{rads}^{-1}(\approx 0.7 \mathrm{~Hz})$. The sign of the inner disk motion was taken as relative to the turntable rotation. These two parameters were controlled by a PC such that either sequences of experiments at fixed parameter values could be performed or slow scans of the parameter space by gradually changing one of the parameters or a suitable combination.

The experiments could either operate using Laser Doppler Anemometry (LDA) to measure the radial velocity component in the centre of the forcing region, or for particle tracking to measure horizontal velocity fields. While the working fluid for the LDA experiments was water, it was a water-glycerol mixture with a density of $\rho=1044 \mathrm{~kg} \mathrm{~m}^{-3}$ to match the density of the tracer particles in the particle tracking experiments, resulting in a kinematic viscosity of $v=1.66 \times 10^{-6} \mathrm{~m}^{2} \mathrm{~s}^{-1}$. The horizontal velocity field in the horizontal plane at mid-level was measured by tracking neutrally buoyant Pliolite particles in the fluid, which were illuminated by a horizontal sheet of light. The light sheet was produced by three projector light bulbs and cylindrical lenses arranged around the periphery of the tank. The horizontal flow was then observed with a monochrome CCD camera, mounted on the turntable and looking vertically down on the tank.

To obtain a representative sample of flow states, three-hour

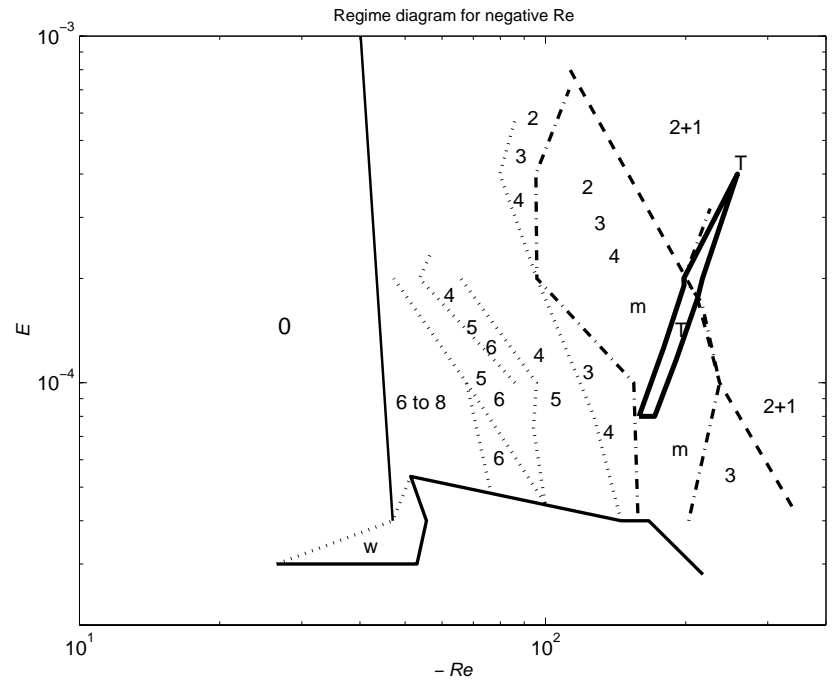

Fig. 3. Regime diagram for the case of the inner disk rotating in the opposite sense of the background rotation (negative $R e$ ). The numbers indicate the number of vortices present, $2+1$ refers to a variety of flows dominated by two unequal vortices. The regimes $\mathrm{m}, \mathrm{T}$, and $\mathrm{w}$ are more complex time-dependent flows. The regime $\mathrm{T}$ is identified by the island enclosed by the thick solid line. The lower solid line indicates the lower limit of Ekman number explored in the experiments. The dashed and dash-dotted lines highlight the regions of two-dimensional time-dependent flows.

periods were recorded onto S-VHS video tapes during which one of the parameters was changed. The examples presented below were obtained by changing $R e$ linearly by a prescribed increment over a short time followed by a longer time where the parameters were held constant. The video tapes were then analysed using the particle tracking option of the flow measurement package DigImage (Dalziel, 1992), which resulted in a file of up to 4096 simultaneous horizontal velocity vectors at the position of identified tracer particles. While the software allowed a calculation of the velocities or derived quantities on a regular grid, the primary velocity data were used to minimize averaging and fitting errors.

With a fluid depth of $H=0.1 \mathrm{~m}$ and a kinematic viscosity of $v=1.66 \times 10^{-6} \mathrm{~m}^{2} \mathrm{~s}^{-1}$, the typical ranges of these parameters were $|R e| \lesssim 3000$ and $E \approx 10^{-5} \ldots 10^{-2}$. It may be noted here, that the Rossby and Reynolds numbers may be positive or negative. If the inner disk is rotating in the same direction as the turntable, $R e$ and $R o$ are positive, and negative otherwise. 


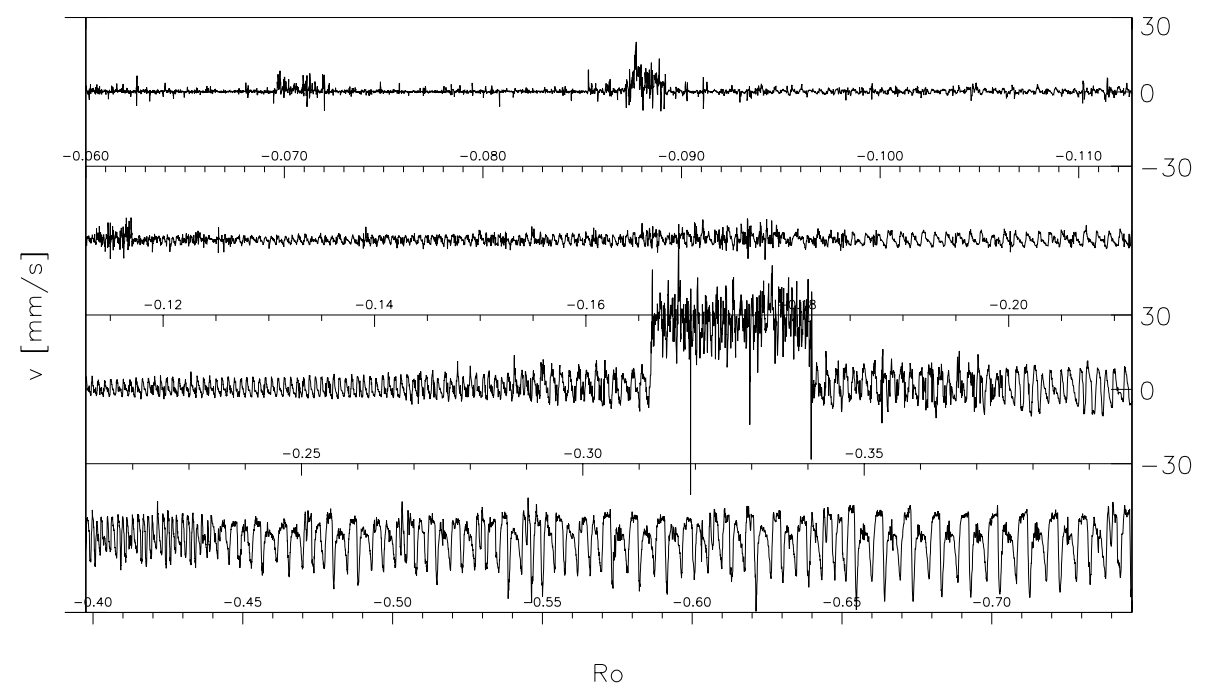

Fig. 4. Time series/bifurcation series at $E=10^{-4}$ showing the radial velocity at the centre of the forcing region as a function of Rossby number which was changed logarithmically over a time interval of 8 hours from $R o=-0.06$ to -0.75 . Due to computer problems, the original data file could not be retrieved to plot it with $R e$ as the $x$-axis. As a result, the figure from a previous analysis had to be reproduced here. The conversion between the two numbers is a $R e=12^{-1 / 4} E^{-3 / 4} R o$. The corresponding Rossby and Reynolds number values at transitions are

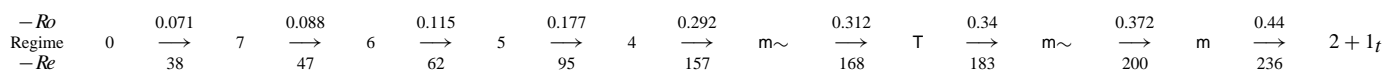

\section{Laboratory results}

\subsection{Overview and steady vortices}

The general findings, as already presented in Früh and Read (1999), can be summarised in two regime diagrams, one for a super-rotating inner disk $(\Delta \Omega, R o, R e>0)$ and another for a sub-rotating inner disk. The regime diagram in Fig. 3 shows the case of a sub-rotating disk $(\Delta \Omega, R o$, Re all negative), which shows essentially the same data as Fig. 4 of Früh and Read (1999) but now transformed from the $E-R o$-plane to the $E-R e$-plane where the definition of the Reynolds number is that of Eq. (11). The regime diagram was obtained from a number of parameter scans using Laser Doppler Anemometry, such as that shown in Fig. 4, while subsequent particle tracking confirmed the regime diagram in the places visited. Figure 4 is a graph of the radial velocity in the centre of the forcing region as a function of the Rossby number, Ro, which was changed linearly over a time interval of about $10 \mathrm{~h}$. As a result, the oscillations visible correspond to temporal oscillations which occurred at the parameter range indicated by the horizontal axes. The plot is subdivided into four panels which form four consecutive parts of a single velocity time series. Mode transitions are visible as sudden changes in the oscillation frequency.

Below a critical Reynolds number of $R e_{c}=36 \pm 3$, the shear layer was stable, denoted by 0 in the regime diagram. When the shear layer became unstable, the flow broke up into a string of regular vortices where the size and number of vortices was mainly determined by the thickness of the layer as measured by the Ekman number. A small Ekman number resulted in a large number of small vortices while a large Ekman number resulted in as few as two vortices. The mode numbers found are indicated by the numbers in Fig. 3 . While it appears that the initial instability is controlled by a single parameter, namely the Reynolds number based on the thickness of the $E^{1 / 4}$-Stewartson layer, the vortex mode transitions are not so easily classified. A comparison of the four main potential candidates - the Ekman number, the two Reynolds numbers, one based on the Stewartson layer thickness the other on the tank dimensions, and the Rossby number - suggests that the transitions are probably largely determined by the Rossby number (cf Figs. 3 and 4 in Früh and Read, 1999). At any given Ekman number, however, the Reynolds numbers and Rossby numbers are related to each other by a fixed conversion factor, and for the purpose of this paper the Reynolds number is used as the second nondimensional parameter; increasing the Reynolds number gradually from the first supercritical vortex flow resulted in a succession of transitions to fewer and larger vortices along the shear layer. Reducing $R e$ from a large value, produced corresponding transitions to more but smaller vortices.

The location of the transitions between different vortex numbers depended on the direction of the change of the experimental conditions. This hysteresis resulted in the coexistence of up to three possible vortex states for any given pair of parameter values. Which of the possible states was observed depended on the initial conditions but, once the 
flow had reached one of the states, it remained in that state for the duration of the experiment. The lowest number of vortices observed was two. All vortex flows with four or more vortices were steady wave-like flows with the appropriate symmetry of the wave number. Only flows with three or two dominant vortices could show some time-dependence or deviations from rotational symmetry. These types of flows are indicated by $m$ and $2+1$, respectively, in Fig. 3 and are discussed in a little more detail in the following section.

Two flow types are not discussed here because it is not expected that they can be modelled as two-dimensional flow. Irregular weak fluctuations, w, were found at the lower limit of the range of Ekman numbers available in the experiment. More surprising is the definite interruption of the vortex flow by the apparently highly irregular or turbulent flow with a large positive mean radial velocity between $R o=-0.31$ and -0.34 in Fig. 4. Despite its crass deviation from the rest of the flow types, this flow, denoted $\mathrm{T}$ in Fig. 3, is highly robust and reproducible. Even more surprising is that it exists in a small band, after which the flow returns to the previous regime. Due to the positive mean radial velocity, the flow appears to involve three-dimensional flow. Comparison with Chomaz et al. (1988) suggested that the flow may be, at least in appearance, similar to a pair of Taylor vortices. However, since this regime is found for a sub-rotating as well as a super-rotating inner disks and cylinder, they cannot be directly equivalent to Taylor vortices since all flows with a slower rotation of the inner disks and cylinders should be stable with respect to the Taylor-Couette instability. Further work to visualise and measure the flow in a vertical cross-section is required to shed some light on this flow phenomenon. At present, we can only note that this flow is characterised by a mean outward flow at mid-height and midradius, and that the transition to this flow is not hysteretic and depends most likely on a Reynolds number whose scaling length is based on the tank geometry rather than the Stewartson layer thickness, e.g. $R e=U H v^{-1}$.

\subsection{Three-vortex flows}

Both a steady and an oscillating three-vortex flow were found, where the oscillating flow is indicated by $\mathrm{m}$ in the regime diagram in Fig. 3. In the example shown in Fig. 4, two forms of the oscillating regime, $\mathrm{m}$ and $\mathrm{m} \sim$ are identified. The only discernible difference between those two types of temporal oscillation is the presence or absence of uncorrelated (white) noise. No physical reason for the noise amplification has yet been found. While the noisy oscillating flow is the most prominent noisy flow found, all other vortex flows showed the tendency to become noisy near mode transitions which may indicate that the basin of attraction of the respective attractor is becoming increasingly 'shallow' as the system approaches the limits of its stable extent.

The flow, $\mathrm{m}$, is consistent with a superposition of a mode 2 and a mode 3 travelling at different speeds along the shear layer, but no particle tracking evidence for this flow exists

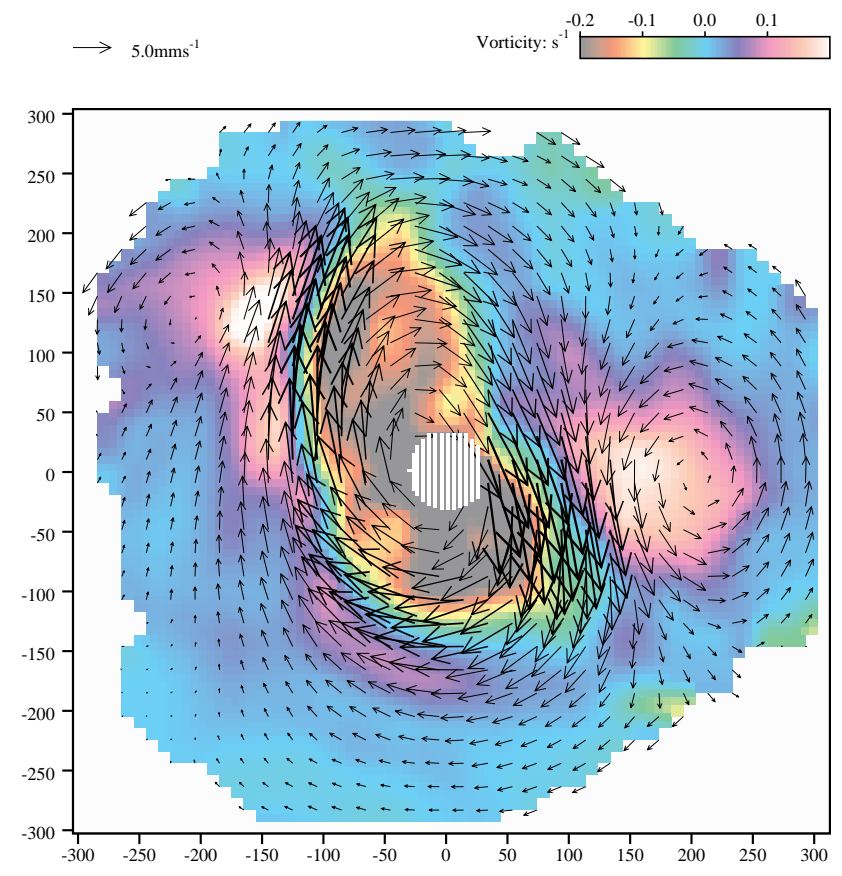

Fig. 5. Velocity and vorticity field from the laboratory experiment of a distorted two-vortex flow at $\operatorname{Re}=-80$ and $E=7.3 \times 10^{-4}$.

yet since the particle tracking experiments concentrated on the steady flows and the two-vortex flows.

\subsection{Two-vortex flows}

A variety of two-vortex flows were found, a steady symmetric flow (indicated by 2), and steady and time-dependent distorted two-vortex flows (both indicated by $2+1$ ). The symmetric flow was found after a mode transition $m=3 \rightarrow 2$ while the other two-vortex flow arose from a gradual distortion of the $m=2$ flow into two vortices of unequal strength and shifted vortex positions. The LDA signal of the steady $2+1$ flow is that of a period-doubled limit cycle as the larger and smaller vortex pass through the measurement point in turn. It can be seen in Fig. 4, that the flow following the transition from $m$ to $2+1$ shows significant variability while it appears to become more regular (or steady) at much higher forcing (e.g. $R o=-0.65$ to -0.72 , or $R e=350$ to 400). In a global mode description, the flow would be a superposition of a mode 1 and a mode 2 . An example of a distorted two-vortex flow is shown in Fig. 5. The arrows represent the horizontal velocity field while the underlying contour map indicates the vorticity. The deformation of the shear region is clearly visible together with the two counter-rotating vortices along the outer edge of the shear layer. The light areas in the superimposed vorticity map indicate low-vorticity regions between the strongest shear and the centre of each vortex. Similar flows were observed in the experiments by Rabaud and Couder (1983) and Chomaz et al. (1988) and in shallow-water experiments in a rotating parabolic vessel by de Konijnenberg et al. (1999). 
Table 2. Physical and nondimensional parameters of PIV sequence at $E=^{-4}$

\begin{tabular}{l|cccc}
\hline Time $(\mathrm{s})$ & $0-720$ & $900-1620$ & $1800-2520$ & $2700-3420$ \\
$\Delta \Omega(\mathrm{rad} / \mathrm{s})$ & -0.159 & -0.136 & -0.114 & -0.0914 \\
$\Omega(\mathrm{rad} / \mathrm{s})$ & 0.599 & 0.576 & 0.554 & 0.531 \\
$R o$ & -0.7 & -0.6 & -0.5 & -0.4 \\
$R e$ & -167 & -143 & -120 & -96 \\
\hline
\end{tabular}

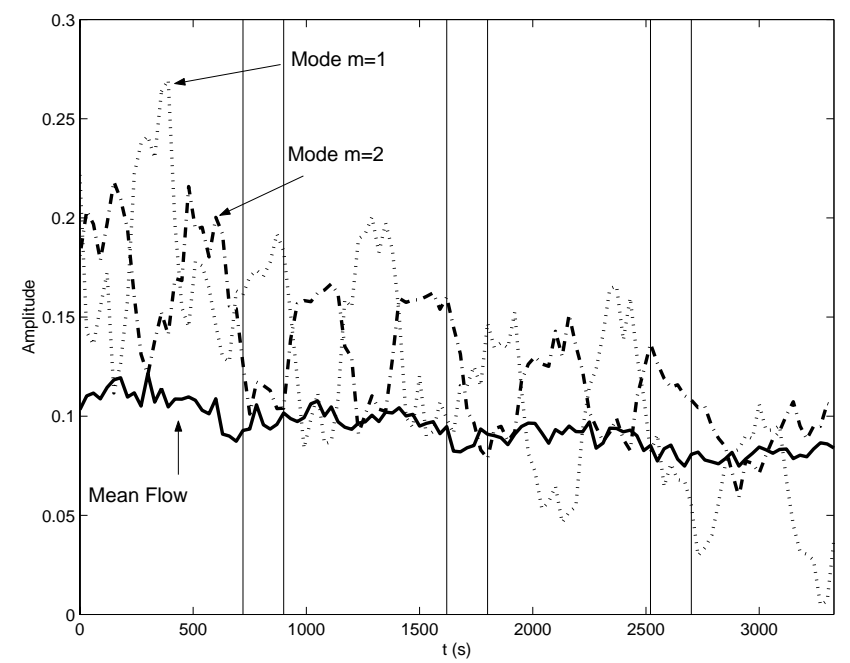

Fig. 6. Time series of empirical modes from an EOF analysis of the set of particle tracking data. The quantities plotted are the amplitude of the first principal component (solid line) and of the two following pairs of principal components in quadrature, representing the azimuthal mean flow, mode $m=2$ (dash-dotted line), and mode $m=1$ (dotted line), respectively.

In the following section a sequence of velocity maps is analysed which starts in the distorted vortex regime and moves in steps towards lower Rossby numbers and the symmetric two-vortex regime. After equilibration, the parameters are held constant for 12 minutes followed by a linear decrease of $R e$ over 3 minutes, after which $R e$ is held constant for 12 minutes once more, and so on. The Ekman number for these flows is $E=7.3 \times 10^{-4}$, and the Reynolds numbers for the constant sections decrease in amplitude from $R e=-167$ to -96 as listed in Table 2. To keep the amount of data at a manageable level, velocity fields were calculated at $30 \mathrm{sec}-$ ond intervals. From the sets of velocity fields, the amplitudes of empirical modes and the position and strength of individual vortices were calculated as outlined in Früh (2002).

Figure 6 is a time series of the amplitudes of empirical modes from an EOF analysis of the velocity fields. More details can be found in Früh (2002) but, in essence, the EOF analysis decomposes a set of velocity fields into empirical orthogonal functions (EOF) which can be regarded as the empirical version of normal modes. In the case of oscillations or drifting waves, two EOFs usually pair up and form a pair in quadrature similar to a sine and cosine pair. The solid line in Fig. 6 shows the first principal component vector which is

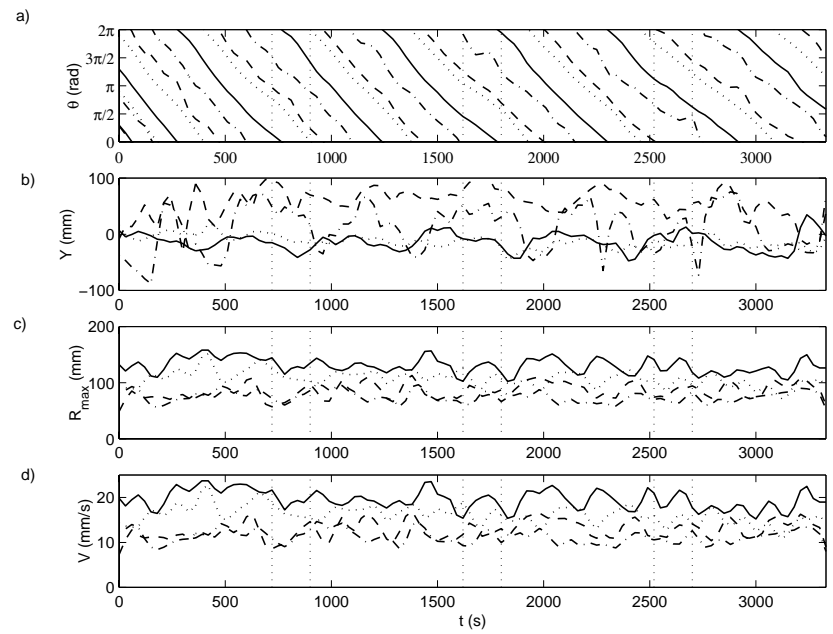

Fig. 7. Time series of the vortex characteristics: (a) Spatial phase of the vortex centres; (b) lateral distance of the vortex centre from the disk edge; (c) major radius of the vortices; and (d) maximum circulation velocity in the vortex. The solid line is for the primary anticyclone, the dotted line for the primary cyclone, the dashed line for the secondary anticyclone, and the dash-dotted line for the secondary cyclone.

the azimuthal mean flow, the amplitude of the first pair which represents a mode 2 , and the amplitude of the following pair, representing a mode 1 , respectively. The amplitudes show a general decrease which reflects the decrease in the energy input with the decrease of the forcing. An oscillation of the two modes in antiphase superimposed on the trend might suggest a mode competition.

In an alternative approach, the time-dependence of the flow was described in terms of the position and strength of individual vortices in the field. Time series of four scalars are shown in Fig. 7, namely the azimuthal position, the radial position, the size, and the strength in panels a) to d) respectively. The position of the vortex is shown by their angle in the cylindrical system of the tank and their radial position as the distance of the core from the edge of the differentially rotating disks, $Y \equiv r-150 \mathrm{~mm}$. The main features are that the four vortices drift on average at the same rate in a retrograde direction, but that the drift of the individual vortices fluctuated, with very little fluctuation in the two primary vortices but pronounced fluctuation in the two secondary vortices. Similar variation was found in the radial position of the vortex centres, which seemed to be locked to the angle. In the plots of the maximum radius ("size"), $R$, and the scaling velocity ("strength"), $V$, of the vortices it becomes apparent that the system gradually moves from one distinct dominant vortex to a state where the differences between the two vortices of same sign gradually reduces. It appears that the size of the vortices is primarily determined by the size of the tank and the total number of vortices present while the velocity scale depends on the forcing by the inner disk rotation as well as the vortex size. The difference between the primary cyclones and anticyclones is a consistent 

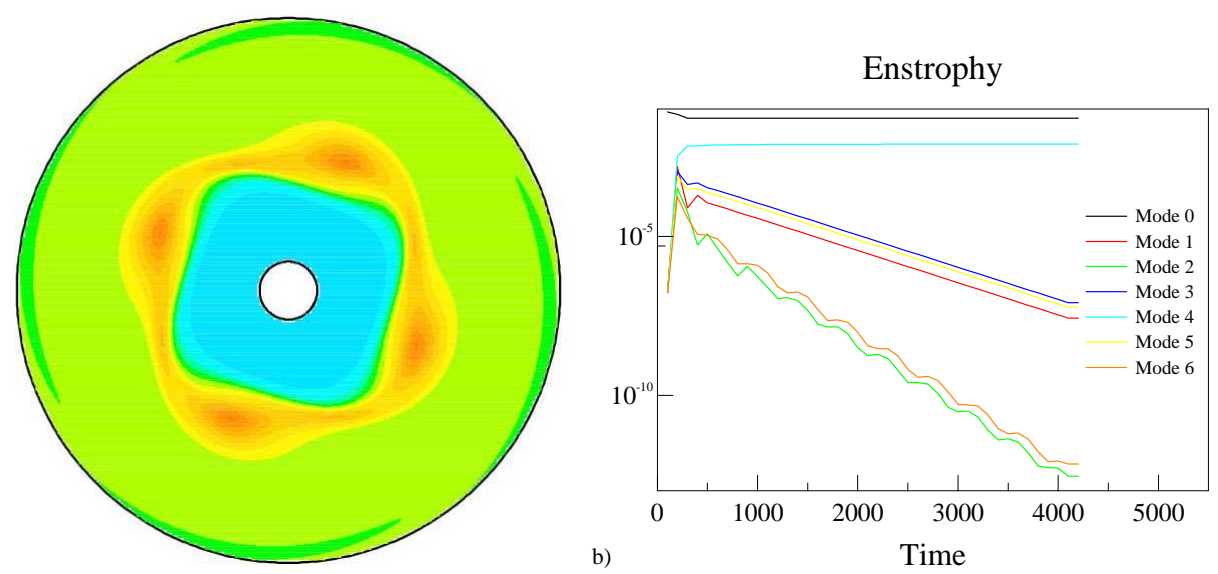

Fig. 8. Equilibrated vorticity field at $R e=99$ and the evolution of the enstrophy contained in the mean flow and first 6 azimuthal waves from initialisation to equilibration.
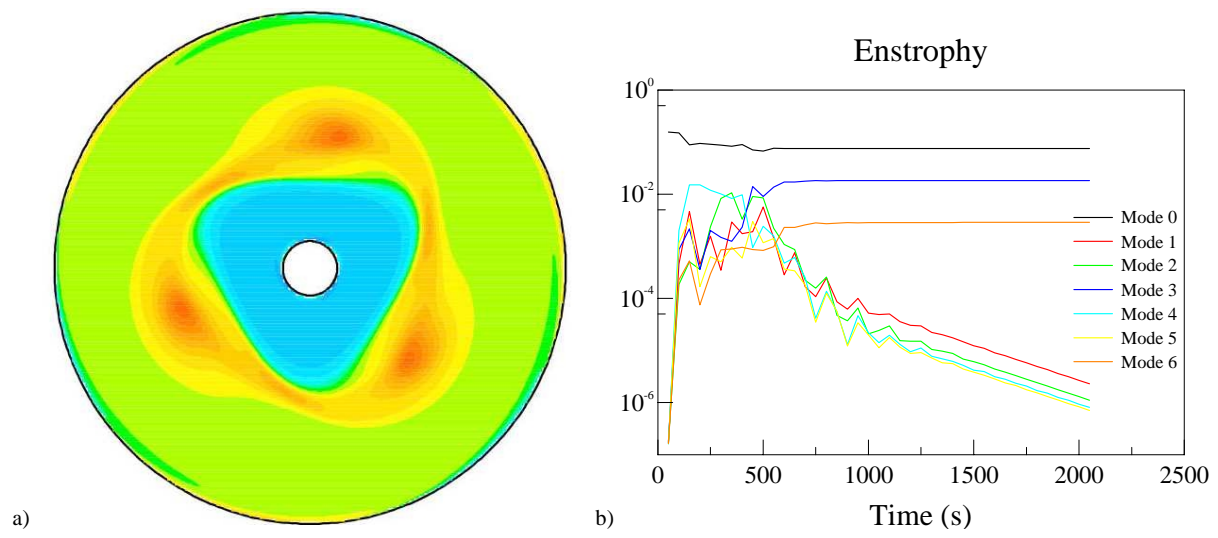

Fig. 9. Equilibrated vorticity field at $R e=138$ and the evolution of the enstrophy contained in the mean flow and first 6 azimuthal waves from initialisation to equilibration.

feature but more work is needed to investigate the reason for this asymmetry. The time scales of fluctuations of $R$ and $V$ seemed to be independent of the spatial phase of the vortices but varied with the forcing. This provides some evidence that time-dependent behaviour may be due to vortex interactions.

\section{Numerical results}

This section will present results from our numerical simulations, using $\delta=9.6 \mathrm{~mm}, H=102.6 \mathrm{~mm}, \Omega=1.5 \mathrm{rad} / \mathrm{s}$ and $v=0.816310^{-6} \mathrm{~m}^{2} / \mathrm{s}$ corresponding to $E=10^{-4}$. This Ekman number showed both types of time-dependency, the mode 3 and 2 oscillation, denoted by $\mathrm{m}$, and the mode 1 and 2 competition within the region indicated by $2+1$ in Fig. 3, respectively. The study explored a range of Reynolds numbers from $R e=99$ to $R e=788$. Consistent with the experiments and other studies, the equilibrated solutions show a sequence of steady vortices with decreasing number of vortices as the Reynolds number increases. The plots show the equilibrated vorticity field at the end of the integration and the tempo- ral evolution of the enstrophy contained in the the azimuthal mean flow and the first six azimuthal wave modes.

At the lowest Reynolds number investigated, $R e=99$ shown in Fig. 8, the flow settled into a mode 4 after only a few seconds, and all other modes decayed rapidly albeit at two different decay rates. Modes 1, 3, and 5 decayed at a fairly steady rate by three to four orders of magnitude over 4000 seconds, while modes 2 and 6 decayed much more rapidly by ten orders of magnitude. The oscillation visible in the latter modes is also present in the others but cannot be seen due to the logarithmic scale of the amplitude axis. In a low-order spectral model of nonlinear baroclinic waves by Früh (1996), it was previously observed that resonant triad interactions involving the long wave, $m=1$, are often the preferred route for energy exchanges between the dominant mode and other modes. If a similar scenario operated in this system, the preferred route for energy or enstrophy away from the dominant mode would be via the triads $(4,1,3)$ and $(4,1,5)$, while the triad $(4,2,6)$ would play a minor role. As a result, we could explain the slower decay of modes $m=1,3$, and 4 , compared to modes $m=2$ and 6 , by the 

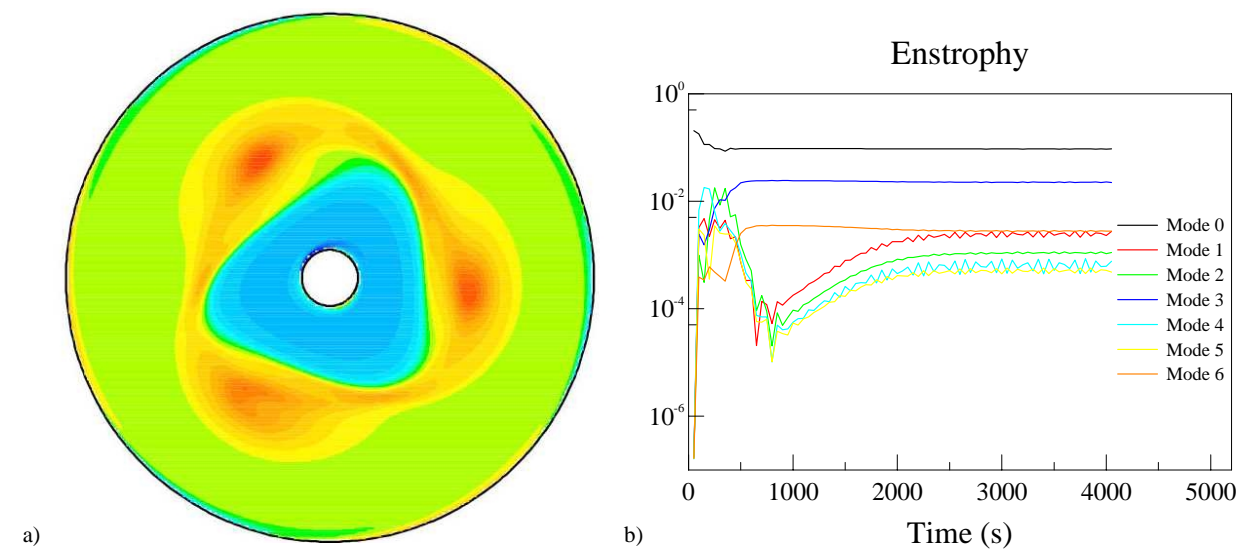

Fig. 10. Equilibrated vorticity field at $R e=156$ and the evolution of the enstrophy contained in the mean flow and first 6 azimuthal waves from initialisation to equilibration.
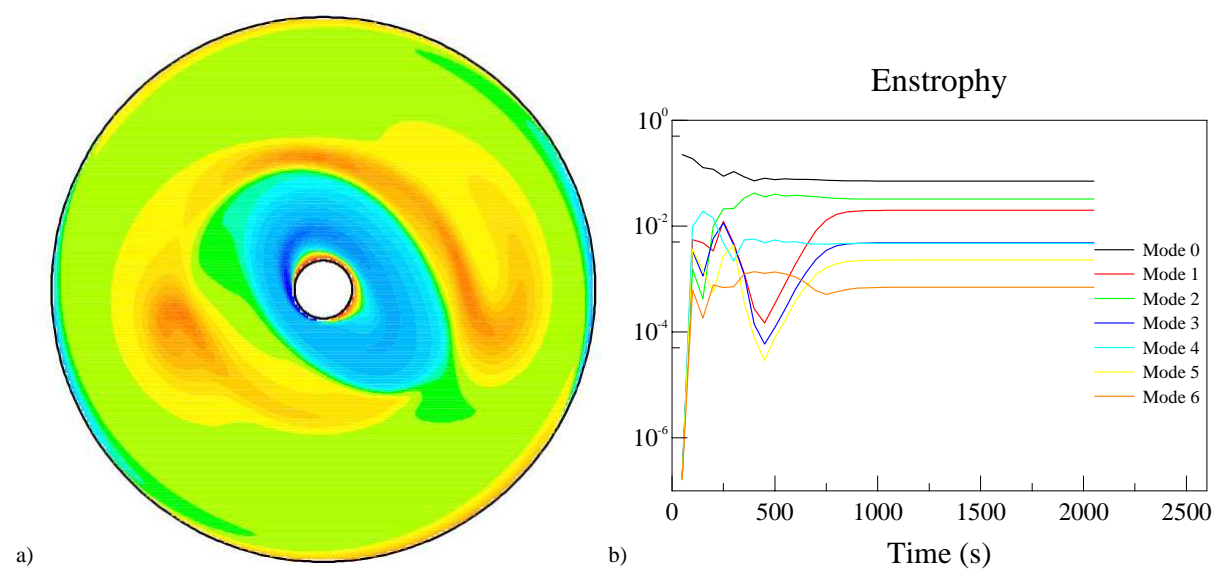

Fig. 11. Equilibrated vorticity field at $R e=166$ and the evolution of the enstrophy contained in the mean flow and first 6 azimuthal waves from initialisation to equilibration.

fact that their decay is slowed down by an enstrophy supply from the dominant mode. Whether such a feature would also be true for the laboratory experiment is still to be confirmed since the laboratory equivalent of the baroclinic waves (Früh and Read, 1997) had revealed that the resonant triads with the long wave were the preferred route only for timedependent waves while the sideband interaction, which includes $(4,2,6)$, was preferred for equilibrated steady waves. This does not, however, preclude that the long-wave triads might be the initial route in the equilibration process.

As the Reynolds number increased, the time for the flow to settle increased noticeably. For $R e>\approx 118$, the flow equilibrated into a mode 3. Close to this Reynolds number, however, the flow settled initially into a mode 4 and changed to three vortices only after a significant transient. At $R e=118$ the transient lasted about 1000 seconds while it had reduced to about 500 seconds at $R e=138$. Once the flow had reached its final number of vortices, the dominant wave number and its harmonics equilibrated to a steady state, as seen in the enstrophy evolution in Fig. 9 while the other modes decayed away.
Increasing the Reynolds number further did not result in a simple transition to two vortices. Instead, the three vortices remained steady but became slightly distorted. This becomes apparent in Fig. 10 for $R e=156$ in the fact that the three lobes in the vorticity field are no longer identical and that all wave modes equilibrated to an almost constant value after the initial transient. It is unclear whether the weak oscillations observable for modes is part of the final solution or a transient.

By $R e=166$, the flow settled into a steady flow with two vortices of unequal strength as shown in Fig. 11 after a relatively short transient. Unlike the case of the distorted mode 3 at $R e=156$, no oscillations in any of the modes are apparent. The steady mode $2+1$ flow persisted over a large range of $R e$ until, at $R e=394$, some weak but regular oscillations of the weaker modes were found. In particular modes 6 and 4 showed clear oscillations while mode 1 showed no discernible oscillation at all. Throughout the $2+1$ regime, the relative amplitude of the modes varied slightly. At $R e=166$, the amplitude of mode 1 compared to mode 2 was $A_{1} / A_{2}=0.62$, which increased to 0.78 at $R e=197$ 
Table 3. Summary of the model integrations presented here

\begin{tabular}{l|cccccccc}
\hline$\Delta \Omega(\mathrm{rad} / \mathrm{s})$ & 0.124 & 0.149 & 0.173 & 0.198 & 0.208 & 0.248 & 0.496 & 0.992 \\
$R e$ & 99 & 118 & 138 & 156 & 166 & 197 & 394 & 788 \\
Flow & 4 & 3 & 3 & $3+1 \sim$ & $2+1$ & $2+1$ & $2+1 \sim$ & $2+1_{t}$ \\
\hline
\end{tabular}
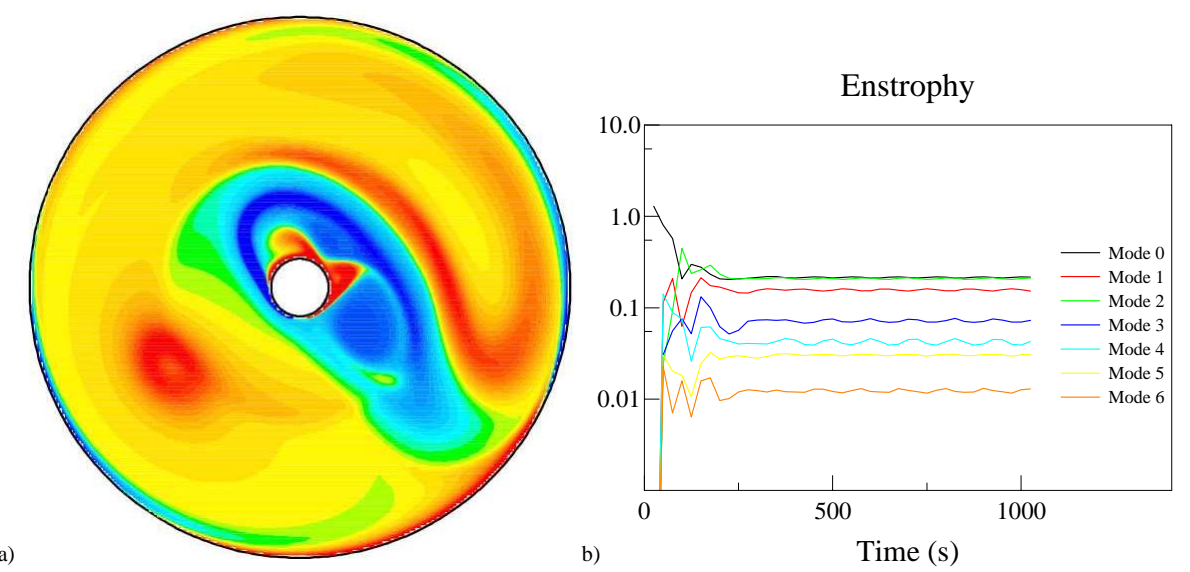

Fig. 12. Equilibrated vorticity field at $R e=394$ and the evolution of the enstrophy contained in the mean flow and first 6 azimuthal waves from initialisation to equilibration.

but then decreased again to 0.69 at $R e=394$. The enstrophy contained in the modes, compared to the mean flow, can be estimated by the amplitude of mode 2 , which increased from $A_{2} / A_{0}=0.45$ at $R e=166$ to 0.50 at $R e=197$ and then decreased again to 0.24 at $R e=394$. This may be an indication that the onset of oscillatory behaviour could be due to an increase in the enstrophy contained by the wave modes. This could lead to an energy and enstrophy transfer from the mean flow and the dominant mode 2 which, at some critical point, may no longer be sustainable.

Time dependence becomes obvious again at a much higher Reynolds number, $R e=788$ in Fig. 13, where the flow field looks like a flow with two main vortices and strong activity at smaller scales. In a description of azimuthal modes, the enstrophy evolution in Fig. 14 shows comparable amplitudes of the mean flow and the first six modes, all of which show strong temporal fluctuations. The fluctuations can be linked to the generation of vortex dipoles at the inner boundary. Dipolar structures, formed as the boundary layer on the inner shaft becomes unstable, are injected into the interior of the domain where they disturb the large-scale mode 2 flow field. This observation is not an artefact of the model resolution, which is relatively high near this boundary anyway, but the vortex generation was also found, and thoroughly tested, by Bergeron et al. (2000) in a different version of the same model.

Returning back to the vorticity field of the more regular flow at $R e=394$ in Fig. 12, it could be argued that the small vortex dipoles near the inner boundary may be a reason for the oscillations. No dipoles can be seen, for example, in the steady two-vortex flow in Fig. 11.

\section{Comparison and conclusions}

The flow regimes found in the experiment at an Ekman number of $E=10^{-4}$ are summarised in Fig. 15 against the Reynolds number as the bifurcation parameter. The vertical line indicates the instability of the Stewartson layer and the horizontal lines show the extent of the existence of steady vortices with their respective mode number. The oscillating lines represent time-dependence of any sort, the presumed interference of modes 2 and 3 in the regime $m$ and the timedependence of the distorted two-vortex flow, indicated by $\left(2+1_{t}\right)$. The interruption of the two-dimensional flow by the presumed Taylor vortices, $\mathrm{T}$, is indicated by the vertical bar. The results from the numerical models are added to the experimental data as the diamonds at their respective mode numbers. A qualitative comparison shows very good agreement between the model results and the majority of the regimes found in the experiment. The model showed an initial instability at a well defined critical Reynolds number to a steady vortex flow with a given mode number. With increasing values of the Reynolds number, the solution underwent a sequence of mode transitions to lower mode numbers, where both the experiment and the model show strong hysteresis. No significant time-dependence is found for flows with mode numbers 4 or larger. The steady distorted twovortex flow and time-dependence for flows involving a mode 2 was found in both, experiment and model, while neither experiment nor model observed a single isolated vortex.

The quantitative comparison between the forcing parameters and resulting flows also shows encouraging agreement. At an Ekman number of $E=10^{-4}$, the regime diagram in 

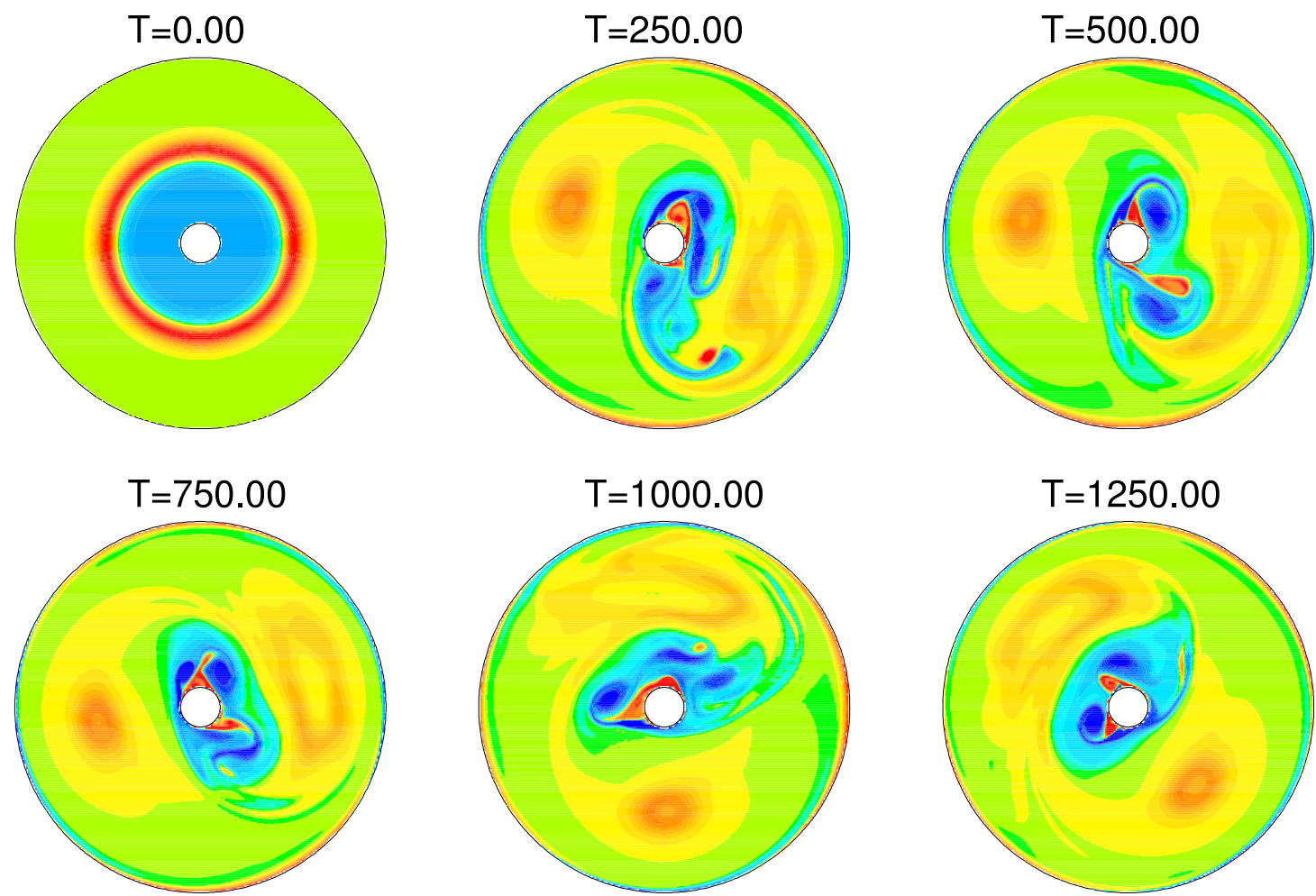

Fig. 13. Vorticity field at $R e=788$ for six time steps starting from initialisation.

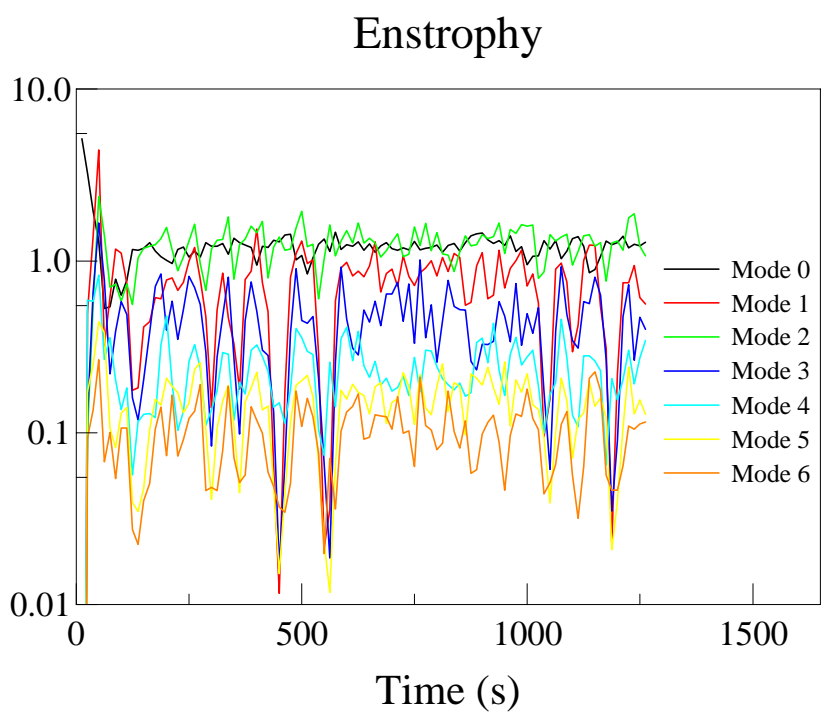

Fig. 14. Evolution of the enstrophy contained in the mean flow and first 6 azimuthal waves from initialisation to equilibration at $R e=788$.

Fig. 3 would predict a mode 4 between $R e \approx 80$ and 120 for the experiments, which is consistent with the model at $R e=99$. Similarly, a mode 3 existed in the experiments between $R e \approx 110$ and 250 , while the model equilibrated to a mode 3 between $R e=118$ and 138 and a weakly oscillating mode 3 at $R e=156$. A mode 2 should exist for $R e>150$ in the experiments, while the numerical model showed a domi- nant mode above $R e=166$.

Despite these agreements, several differences were observed. While the experiment showed only symmetric mode 3 flows (with the experimental uncertainty) and both symmetric and distorted mode 2 flows, the numerical model showed symmetric and weakly distorted mode 3 flows but only distorted mode 2 flows. Furthermore, the oscillation, $\mathrm{m}$, in the experiments which might be a mode 3 and 2 interference was not reproduced in the present model set-up even though the same model adapted to the free-surface parabolic annulus did show a vacillating mode 3 (cf Fig. 21 in de Konijnenberg et al., 1999). It is possible that the weak oscillations apparent in Fig. 10 are an indication of a vacillating mode 3 in the vicinity of that run. However, it seems that the bifurcation from a steady mode 3 to an oscillatory mode 3 is preceded in the current model set-up by the bifurcation from the steady and symmetric mode 3 to a steady but distorted mode 3 . The regime of weak fluctuations, w, was not reproduced but also not expected since it occurs at much smaller Ekman numbers, and it is doubtful whether this regime shows two-dimensional flows anyway. The three-dimensional flow of possible Taylor vortices, T, could obviously not be reproduced by the two-dimensional model.

The mode 1 and 2 competition, seen in Fig. 6 and Fig. 7 in the experiment, and in Fig. 13 and Fig. 14 in the model, show some similarities in that the flows are dominated by the mode 2, together with substantial amplitude in mode 1 and the higher modes. The oscillatory behaviour, however, is somewhat different between experiment and model. In the 


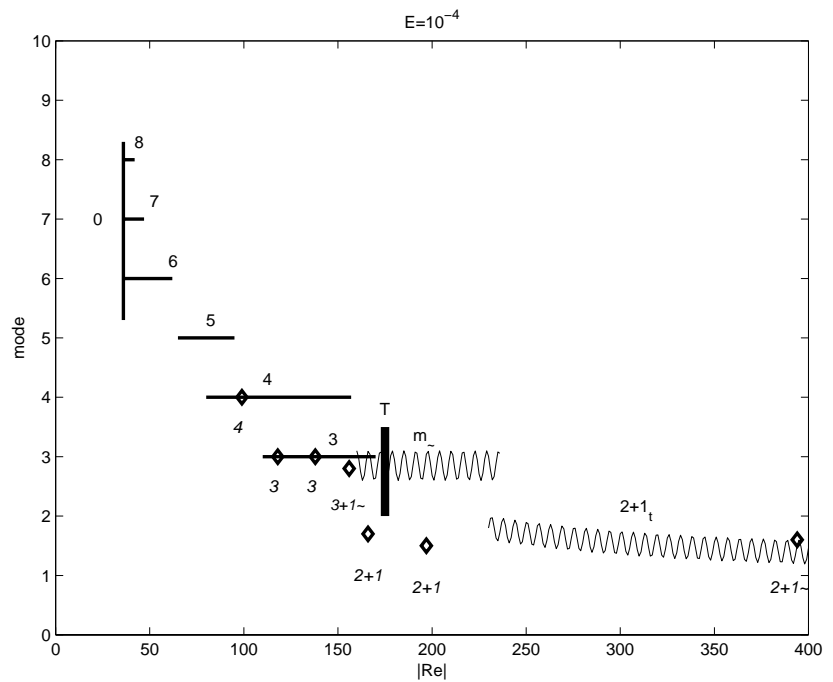

Fig. 15. Scenario of instability, mode transitions, mixed-mode flows, and time-dependence for the experimental results at $E=$ $10^{-4}$ with the numerical results added as the diamonds. The interruption of the two-dimensional flow by the presumed Taylor vortices is indicated by the vertical bar. The labels above the lines refer to the experimental data and the labels below the diamonds refer to the model integrations

experiments, the fluctuations of modes 2 and 1 were on a time scale of around 200s, and the fluctuations of both modes was around a similar mean value. In the model, however, the time scale was only around 100s, and the mean of mode 2 is substantially larger than that of mode 1 . Referring to the motion of vortices, in Fig. 7, and the sequence of vorticity fields in Fig. 13 highlights the similarities again. In the numerical model, the fluctuations appeared to be caused by the generation of vortices at the inner boundary which are injected into the interior of the domain and strongly interfered with the large-scale two-vortex pattern. In the experimental analysis, which was restricted to always showing the position of the four most prominent vortices, the time variation was strongest in the lateral position of the weaker vortices. One explanation could be that such vortices did indeed oscillate in the radial direction, but it could also reflect the generation of such a vortex near the inner boundary and its subsequent drift outwards, where the apparent return of the vortex could be an artifact of the analysis which insisted on the continuing existence of the vortices. The Reynolds number at which the respective flows are found vary are, $R e>\approx 200$ in the experiments and $R e=788$ in the numerical model which showed a steady $2+1$ at $R e=197$.

\subsection{Summary}

The findings from both experiment and model may be summarised by the bifurcation scenario laid out in Fig. 16 with increasing Reynolds number: Following the initial instability of the Stewartson layer, the flow equilibrates to a steady vortex flow characterised by a single mode and its higher har-

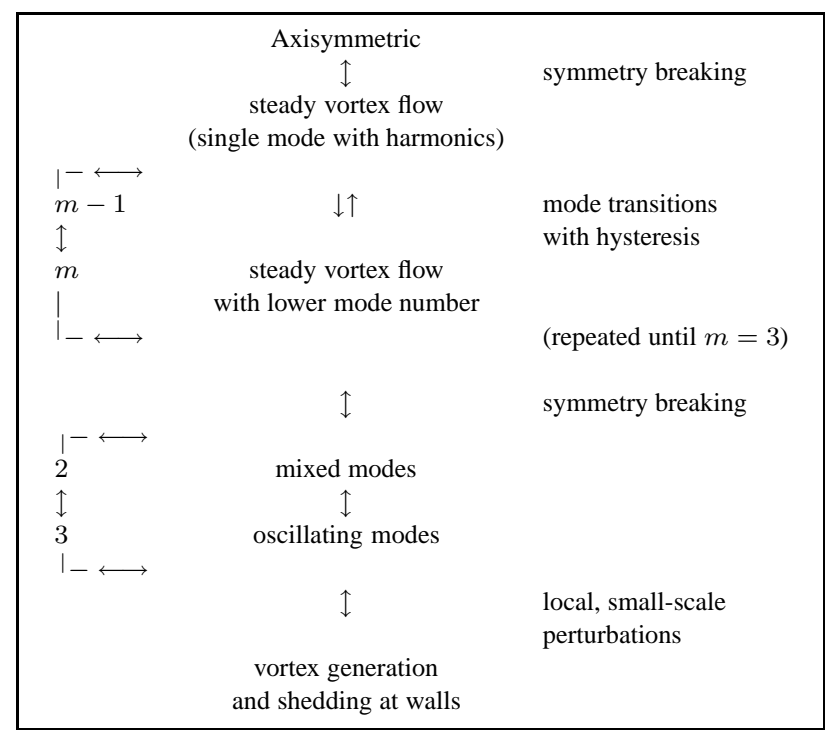

Fig. 16. Schematic bifurcation scenario for the two-dimensional shear layer system.

monics. The flow then undergoes a sequence of mode transitions, each with substantial hysteresis.

More complex flows are characterised by the co-existence of modes. It is possible to identify three common types of mixed-mode flows. If the modes all move at the same velocity, then the resulting flow is a steady vortex pattern of unequal vortices with the symmetry of the lowest common denominator. An example is the steady distorted two-vortex flow found in the model. A linear superposition of two modes drifting at different phase velocities leads to an interference vacillation, which might be the flow found in the experiment and termed $\mathrm{m}$ in the regime diagram. Such a flow is possible without nonlinear interactions between the modes, while the steady mixed-mode flow generally requires non-linear interactions to cause the phase-locking of the different modes. Finally, a mode competition involving significant nonlinear interactions, may lead to more complex flows, such as amplitude vacillations. It will depend on parameters other than the Reynolds number which type of mixed-mode flow actually occurs. The experimental regime diagram suggests that it would be worthwhile to explore the dependence of the transitions on the Ekman number in the model, which would be done by choosing appropriate values of the forcing length, $\delta_{F}$. The differences between the experiment and model may be due to the current choice of the forcing length, $\delta_{F}$, in the model and the subsequent definition of $E$ and $R e$. The model has been shown by de Konijnenberg et al. (1999) to be able to generate oscillating state involving mode 2 and 3 , which may be an interference vacillation or amplitude vacillation suggested here.

The final transition observed in the model is better understood by the interactions of local dynamics with the largescale modes. This is the generation of vortex dipoles at the wall which are then moving into the fluid interior and interact 
with the large-scale modal flow patterns. The description of such flow in terms of global modes requires the inclusion of a large number of modes and results in highly irregular temporal fluctuations at large amplitudes off all modes. This flow characterises the limit of low-order nonlinear dynamics for this system. It should be noted that the irregular regime, T, which may correspond to Taylor vortices, found consistently in the experiment is omitted in this broad brush summary.

To conclude, it appears that two very different processes in the shear layer system may lead to time dependency. One process is the co-existence of global modes, with or without nonlinear interactions between modes being active. This process may occur independently of the side boundary conditions. The other process involves a local phenomenon, the generation of small vortices at boundaries which then interact with the large-scale flow.

Acknowledgements. This research arose from a research project headed by Dr Peter Read and funded by the Natural Environment Research Council of the UK. The collaboration between the authors is the result of a visit to Ris $\varnothing$ National Laboratory and the University of Copenhagen whose financial support are gratefully acknowledged.

\section{References}

Antipov, S. V., Nezlin, M. V., Snezhkin, E. N., and Trubnikov, A. S.: Rossby autosoliton and stationary model of the Jovian Great Red Spot, Nature, 323, 238-240, 1986.

Baker, J. D.: Shear layers in a rotating fluid, J. Fluid Mech., 29, 165-175, 1967.

Bergeron, K., Coutsias, E. A., Lynov, J. P., and Nielsen, A. H.: Dynamical properties of forced shear layers in an annular geometry, J. Fluid Mech., 402, 255-289, 2000.

Chomaz, J. M., Rabaud, M., Basdevant, C., and Couder, Y.: Experimental and numerical investigation of a forced circular shear layer, J. Fluid Mech., 187, 115-140, 1988.

Dalziel, S. B.: Decay of rotating turbulence: some particle tracking experiments, J. Appl. Sci. Res., 49, 217-244, 1992.

de Konijnenberg, J. A. V., Nielsen, A. H., Rasmussen, J. J., and Stenum, B.: Shear-flow instability in a rotating fluid, J. Fluid
Mech., 387, 177-204, 1999.

Früh, W.-G.: Low-order models of wave interactions in the transition to baroclinic chaos, Nonlin. Proc. Geophys., 3, 150-165, 1996.

Früh, W.-G.: Methods to describe barotropic vortices by global fields and vortex characteristics, Nonlin. Proc. Geophys., 9, 189200, 2002.

Früh, W.-G. and Read, P. L.: Wave interactions and the transition to chaos of baroclinic waves in a thermally driven rotating annulus, Phil. Trans. R. Soc. Lond. (A), 355, 101-153, 1997.

Früh, W.-G. and Read, P. L.: Experiments in a barotropic rotating shear layer. I: Instability and steady vortices, J. Fluid Mech., 383, 143-173, 1999.

Greenspan, H. P.: The theory of rotating fluids, Cambridge University Press, Cambridge, 1968.

Hide, R. and Titman, C. W.: Detached shear layers in a rotating fluid, J. Fluid Mech., 29, 39-60, 1967.

Holton, J. R.: An experimental study of forced barotropic Rossby waves, Geophys. Fluid Dyn., 2, 323-341, 1971.

Humphrey, J. A. C. and Gor, D.: Experimental observations of an unsteady detached shear layer in enclosed corotating disk flow, Phys. Fluids, A 5, 2438-2442, 1993.

Meyers, S. D., Sommeria, J., and Swinney, H. L.: Laboratory study of the dynamics of Jovian-type vortices, Physica D, 37, 515-530, 1989.

Nezlin, M. V., Rylov, A. Y., Trubnikov, A. S., and Khutorestkiï, A. V.: Cyclonic-anticyclonic asymmetry and a new soliton concept for Rossby vortices in the laboratory, oceans and the atmospheres of giant planets, Geophys. Astrophys. Fluid Dyn., 52, 211-247, 1990.

Niino, H. and Misawa, N.: An experimental and theoretical study of barotropic instability, J. Atmos. Sci., 41, 1992-2011, 1984.

Pedlosky, J.: Geophysical Fluid Dynamics, Springer-Verlag, Berlin, Heidelberg, New York, second edn., 1987.

Rabaud, M. and Couder, Y.: A shear-flow instability in a circular geometry, J. Fluid Mech., 136, 219-319, 1983.

Schär, C. and Davies, H. C.: An instability of mature cold fronts, J. Atmos. Sci., 47, 929-950, 1990.

Solomon, T. H., Holloway, W. J., and Swinney, H. L.: Shear flow instabilities and Rossby waves in barotropic flow in a rotating annulus, Phys. Fluids A, 5, 1971-1982, 1993.

Stewartson, K.: On almost rigid rotations, J. Fluid Mech., 3, 17-26, 1957. 